\title{
Asymptotically Good Convolutional Codes
}

\author{
Giuliano G. La Guardia *
}

March 29, 2018

\begin{abstract}
In this paper, we construct new sequences of asymptotically good convolutional codes (AGCC's). These sequences are obtained from sequences of transitive, self-orthogonal and self-dual algebraic geometry (AG) codes attaining the Tsfasman-Vladut-Zink bound. Furthermore, by applying the techniques of expanding, extending, puncturing, direct sum, the $\langle u \mid u+v\rangle$ construction and the product code construction to these block codes, we construct more new sequences of asymptotically good convolutional codes. Additionally, we show that the new constructions presented here also hold when applied to al 1 sequences of asymptotically good block codes where $\lim _{j \rightarrow \infty} k_{j} / n_{j}$ and $\lim _{j \rightarrow \infty} d_{j} / n_{j}$ exist.
\end{abstract}

Index Terms - convolutional codes, transitive codes, code construction

\section{Introduction}

In his seminal work [10, Goppa introduced the well-known class of algebraic geometry (AG) codes. It is well known that the AG codes are asymptotically good; because of this fact, coding theorists have utilized this structure in order to obtain (maximal) curves over which more families of asymptotically good codes can be constructed (see [10, 11, 6, 7, 35]). More information with respect to the class of AG codes can be found in [36].

Concerning the investigation and development of theory of convolutional codes, much effort has been paid [5, 31, 32, 33, 13, 8, 9, 14, 17, 28. More specifically, constructions of convolutional codes with good or even optimal parameters (for instance, maximum-distance-separable (MDS) codes, i.e., codes attaining the generalized Singleton bound [32]) are of great interest for several researchers [32, 33, 13, 8, 18, 19, 20.

In this context, constructions of families of good or asymptotically good convolutional codes (AGCC) are also of great importance in the literature [16, 29, 34, 24, 21, 37, 26, 27, 25, 2]. In [16], Justesen constructed AGCC

\footnotetext{
${ }^{*}$ Giuliano Gadioli La Guardia is with Department of Mathematics and Statistics, State University of Ponta Grossa (UEPG), 84030-900, Ponta Grossa, PR, Brazil.
} 
generated by generator polynomials of cyclic codes. In [29, the authors utilized a different approach to design good convolutional codes. More precisely, the authors assume no restrictions in the rate of the code, but only restrictions in the constraint length. Using this approach, they found families of AGCC's with applications in CDMA systems. In [34, the authors introduced an ensemble of $(J ; K)$-regular LDPC convolutional codes and they presented lower bounds on the free distance of such codes. After this, they showed that the ratio between the free distance and the constraint length is several times larger than the ratio of minimum distance for Gallagers ensemble of $(J ; K)$-regular LDPC block codes. In 24, the authors computed a lower bound on the free distance for several ensembles of asymptotically good protograph-based lowdensity parity-check (LDPC) convolutional codes. They utilized ensembles of LDPC convolutional codes (which were introduced by Felstrom and Zigangirov in [4) derived from a protograph-based ensemble of LDPC block codes to obtain asymptotically good, periodically time-varying LDPC convolutional code ensembles, having the property that the free distance grows linearly with constraint length. In [21], the authors performed an iterative decoding threshold analysis of LDPC block code ensembles formed by certain classes of LDPC convolutional codes. These ensembles were shown to have minimum distance growing linearly with block length and their thresholds approach the Shannon limit as the termination factor tends to infinity. In [37, Uchikawa et al. generalized the results shown in 4 to non-binary LDPC convolutional codes. They also investigated its rate-compatibility. In particular, they modified the construction method proposed in 4], to construct a non-binary $(2,4)$-regular LDPC convolutional code. Applying numerical simulations they showed that non-binary rate $1 / 2$ LDPC convolutional codes binary LDPC convolutional codes with comparable constraint bit length. Mitchell et al. [26] showed that several ensembles of regular and irregular LDPC convolutional codes derived from protograph-based LDPC block codes are asymptotically good. Furthermore, they investigated the trapping set (see the definition in 22]) of such class of codes. Mu et al. [27] constructed time-varying convolutional low-density parity check (LDPC) codes derived from block LDPC codes based on improved progressive edge growth (PEG) method (see [3]). Different from the conventional PEG algorithm, the parity-check matrix is initialized by inserting certain patterns. Applying simulation results the authors showed that the new convolutional codes perform well over additive white Gaussian noise(AWGN) channels. Mitchell et al. 25] investigated relationship between the minimum distance growth rate of the spatially coupled LDPC block codes (SC-LDPC-BC) ensemble and the free distance growth rate of the associated spatially coupled LDPC convolutional codes (SCLDPC-CC) ensemble. They showed that the minimum distance growth rates converge to a bound on the free distance growth rate of the unterminated SCLDPC-CC ensemble. Bocharova et al. 2] proposed an interesting search for good convolutional LDPC codes over binary as well as non-binary alphabets by means of three algorithms. They presented examples of codes with bi-diagonal structure of the corresponding parity-check matrix, which preserves low encoding complexity. 
In great part of the works mentioned above, the authors considered timevarying convolutional codes, whereas, in our case, we construct convolutional codes whose (reduced basic) generator matrices do not depend on the time.

In 32, the authors introduced the generalized Singleton bound: if $C$ is an $\left(n, k, \gamma ; m, d_{f}\right)_{q}$ convolutional code then $d_{f} \leq(n-k)[\lfloor\gamma / k\rfloor+1]+\gamma+1$, where $n$ denotes the length, $k$ is the dimension, $\gamma$ is the degree of the code, $m=\max _{1 \leq i \leq k}\left\{\gamma_{i}\right\}$ is the memory and $d_{f}=\operatorname{wt}(V)=\min \{w t(\mathbf{v}(D)) \mid \mathbf{v}(D) \in$ $V, \mathbf{v}(D) \neq 0\}$ denotes the free distance of the code. This upper bound clearly generalizes the Singleton bound ( $\gamma=0$ for block codes).

Based on the generalized Singleton bound, given a sequence $\left(V_{j}\right)_{j \geq 0}$ of convolutional codes with parameters $\left(n_{j}, k_{j}, \gamma_{j} ; m_{j},\left(d_{f}\right)_{j}\right)_{q}$, we present the first contribution of this paper, that is, we introduce the quantities $s_{j}:=\left(n_{j}-\right.$ $\left.k_{j}\right)\left[\left\lfloor\gamma_{j} / k_{j}\right\rfloor+1\right]+\gamma_{j}+1$ and $r_{j}:=\max \left\{n_{j}, s_{j}\right\}$. These two quantities allow us to define more naturally the concept of AGCC in the following sense (see Definition 3.1). Considering the sequence $\left(V_{j}\right)_{j \geq 0}$ of convolutional codes given above, if $\limsup _{j \rightarrow \infty} k_{j} / n_{j}>0$ and if $\limsup _{j \rightarrow \infty}\left(d_{f}\right)_{j} / r_{j}>0$ hold, where $s_{j}:=\left(n_{j}-k_{j}\right)\left[\left\lfloor\gamma_{j} / k_{j}\right\rfloor+1\right]+\gamma_{j}+1$ and $r_{j}:=\max \left\{n_{j}, s_{j}\right\}$, then one says that the sequence is asymptotically good. On the other hand one says that a sequence $\left(C_{j}\right)_{j \geq 0}$ of linear block codes over $\mathbb{F}_{q}$ with parameters $\left[n_{j}, k_{j}, d_{j}\right]_{q}$ is asymptotically good if $n_{j} \rightarrow \infty$ as $j \rightarrow \infty$ and if $\limsup _{j \rightarrow \infty} k_{j} / n_{j}>0$ and $\lim \sup _{j \rightarrow \infty} d_{j} / n_{j}>0$ are true. Thus, these two definitions are analogous in the sense that they consider a comparison between length and dimension as well as they provide a comparison between the free distance and the quantity $r_{j}$, which is the maximum between $n_{j}$ and the Singleton bound $s_{j}$ (note that the consideration of $s_{j}$ in the case of convolutional codes is natural, because $s_{j}$ could be greater than $n_{j}$ ).

As the second contribution, starting from transitive AG codes in 35, we construct new families of convolutional codes having reduced basic generator matrices by utilizing Piret's technique [30. After this, we show that these new families of convolutional codes are asymptotically good. An advantage of our method is that it is performed algebraically. More precisely, given a family of asymptotically good block codes with given parameters (in particular, the transitive codes shown in this paper), our technique allows us to construct the corresponding family of asymptotically good convolutional codes and also to compute the exact parameters of these codes (except the free distance, where a lower bound is given). Additionally, we do not utilize algorithms nor computational search to this end.

The third contribution of this work is based on the second one: starting from techniques of code expansion, extension, direct sum, the $\langle u \mid u+v\rangle$ construction, puncturing and direct product construction applied to such AG codes, we carefully construct sequences of convolutional codes with reduced basic generator matrices, after showing that such families are good asymptotically. Moreover, we show that our proposed constructions also hold when applied to all sequences of asymptotically good block codes where $\lim _{j \rightarrow \infty} k_{j} / n_{j}$ and $\lim _{j \rightarrow \infty} d_{j} / n_{j}$ exist.

Another well-known class of asymptotically good codes is the class of ran- 
dom binary and non-binary convolutional codes with low-density parity-check matrices (see [37, 27]). Since such class of convolutional codes is asymptotically good, it is interesting to know how to relate the free distances of these codes with the ones presented in this paper. However, because random convolutional LDPC codes are often constructed by means of algorithms or they are found by computational search, and the new asymptotically good convolutional codes presented here are constructed algebraically, without using algorithms or even without using computational search, it is difficult to compare their free distances for fixed block lengths. In other words, due to the big difference between both construction methods we cannot perform a comparison among such codes.

The paper is organized as follows. In Section 2, we review the concepts on convolutional codes. In Section 3 we establish the contributions of this work, i.e., constructions of new sequences of asymptotically good convolutional codes. Finally, in Section 4 the final considerations are drawn.

\section{Review of Convolutional Codes}

In this section we present a brief review of convolutional codes. For more details we refer the reader to [5, 15, 12.

Throughout this paper, $q$ is a prime power and $\mathbb{F}_{q}$ is the finite field with

$q$ elements. Recall that a polynomial encoder matrix $G(D) \in \mathbb{F}_{q}[D]^{k \times n}$ is called basic if $G(D)$ has a polynomial right inverse. A basic generator matrix is called reduced (or minimal [33, 12]) if the overall constraint length $\gamma=\sum_{i=1}^{k} \gamma_{i}$, where $\gamma_{i}=\max _{1 \leq j \leq n}\left\{\operatorname{deg} g_{i j}\right\}$, has the smallest value among all basic generator matrices (in this case the overall constraint length $\gamma$ will be called the degree of the resulting code).

Definition 2.1 [15] A rate $k / n$ convolutional code $V$ with parameters $(n, k, \gamma$; $\left.m, d_{f}\right)_{q}$ is a submodule of $\mathbb{F}_{q}[D]^{n}$ generated by a reduced basic matrix $G(D)=$ $\left(g_{i j}\right) \in \mathbb{F}_{q}[D]^{k \times n}$, i.e. $V=\left\{\mathbf{u}(D) G(D) \mid \mathbf{u}(D) \in \mathbb{F}_{q}[D]^{k}\right\}$, where $n$ is the length, $k$ is the dimension, $\gamma$ is the degree, $m=\max _{1<i<k}\left\{\gamma_{i}\right\}$ is the memory and $d_{f}=w t(V)=\min \{w t(\mathbf{v}(D)) \mid \mathbf{v}(D) \in V, \mathbf{v}(D) \neq 0\}$ is the free distance of the code.

In the above definition, the weight of an element $\mathbf{v}(D) \in \mathbb{F}_{q}[D]^{n}$ is defined as $\operatorname{wt}(\mathbf{v}(D))=\sum_{i=1}^{n} \operatorname{wt}\left(v_{i}(D)\right)$, where $\operatorname{wt}\left(v_{i}(D)\right)$ is the number of nonzero coefficients of $v_{i}(D)$. Let us consider the field of Laurent series $\mathbb{F}_{q}((D))$, whose elements are given by $\mathbf{u}(D)=\sum_{i} u_{i} D^{i}$, where $u_{i} \in \mathbb{F}_{q}$ and $u_{i}=0$ for $i \leq r$, for some $r \in \mathbb{Z}$. The weight of $\mathbf{u}(D)$ is defined as $\operatorname{wt}(\mathbf{u}(D))=\sum_{\mathbb{Z}} \operatorname{wt}\left(u_{i}\right)$. A generator matrix $G(D)$ is called catastrophic if there exists a $\mathbf{u}(D)^{k} \in \mathbb{F}_{q}((D))^{k}$ of infinite Hamming weight such that $\mathbf{u}(D)^{k} G(D)$ has finite Hamming weight. 
Since a basic generator matrix is non-catastrophic, all the convolutional codes constructed in this paper have non-catastrophic generator matrices.

We next recall how to construct a convolutional code derived from a block code. This technique was presented first by Piret [30, 31 to binary codes, after generalized by Aly et al. 1] to nonbinary alphabets.

Let $C$ be an $[n, k, d]_{q}$ linear block code with parity check matrix $H$. We split $H$ into $m+1$ disjoint submatrices $H_{i}$ such that

$$
H=\left[\begin{array}{c}
H_{0} \\
H_{1} \\
\vdots \\
H_{m}
\end{array}\right],
$$

where each $H_{i}$ has $n$ columns, obtaining the polynomial matrix

$$
G(D)=\tilde{H}_{0}+\tilde{H}_{1} D+\tilde{H}_{2} D^{2}+\ldots+\tilde{H}_{m} D^{m},
$$

where the matrices $\tilde{H}_{i}$, for all $1 \leq i \leq m$, are derived from the respective matrices $H_{i}$ by adding zero-rows at the bottom in such a way that the matrix $\tilde{H}_{i}$ has $\kappa$ rows in total, where $\kappa$ is the maximal number of rows among the matrices $H_{i}$. The matrix $G(D)$ generates a convolutional code with $\kappa$ rows and memory $m$. In this context, one has the following result:

Theorem 2.1 [1, Theorem 3] Suppose that $C \subseteq \mathbb{F}_{q}^{n}$ is a linear code with parameters $[n, k, d]_{q}$ and assume also that $H \in \mathbb{F}_{q}^{(n-k) \times n}$ is a parity check matrix for $C$ partitioned into submatrices $H_{0}, H_{1}, \ldots, H_{m}$ as in Eq. (1) such that $\kappa=\operatorname{rk} H_{0}$ and $\operatorname{rk} H_{i} \leq \kappa$ for $1 \leq i \leq m$, where $\operatorname{rk} H_{i}$ denotes the row rank of the matrix $H_{i}$. Consider the polynomial matrix $G(D)$ as in Eq. (2). Then the matrix $G(D)$ is a reduced basic generator matrix of a convolutional code $V$. Additionally, if $d_{f}$ denotes the free distances of $V$ and $d^{\perp}$ is the minimum distance of $C^{\perp}$, then $d_{f} \geq d^{\perp}$.

\section{Asymptotically Good Convolutional Codes}

In this section we present the contributions of this paper. More precisely, we construct new sequences of asymptotically good convolutional codes.

First, we recall some basic concepts necessary for the development of this paper. A subgroup $H$ of the symmetric group $S_{n}$ is called transitive if for any pair $(i, j)$ with $i, j \in\{1, \ldots, n\}$, there exists a permutation $\rho \in H$ such that $\rho(i)=j$. A permutation $\rho \in S_{n}$ is called an automorphism of the code $C \subseteq \mathbb{F}_{n}^{q}$ if $\left(c_{1}, \ldots, c_{n}\right) \in C \Rightarrow\left(c_{\rho(1)}, \ldots, c_{\rho(n)}\right) \in C$ holds for all codewords $\left(c_{1}, \ldots, c_{n}\right) \in C$. The group $\operatorname{Aut}(C) \subseteq S_{n}$ is the group of all automorphisms of $C$. A code $C$ over $\mathbb{F}_{q}$ of length $n$ is called transitive if its automorphism group $\operatorname{Aut}(C)$ is a transitive group of $S_{n}$. An example of transitive codes are the well-known cyclic codes. 
Recall that the generalized Singleton bound [33] of an $\left(n, k, \gamma ; m, d_{f}\right)_{q}$ convolutional code is given by $d_{f} \leq(n-k)[\lfloor\gamma / k\rfloor+1]+\gamma+1$. We put $s:=$ $(n-k)[\lfloor\gamma / k\rfloor+1]+\gamma+1$. Based on this upper bound for $d_{f}$, we can introduce more precisely the concept of asymptotically good convolutional codes.

Definition 3.1 A sequence $\left(V_{j}\right)_{j \geq 0}$ of convolutional codes with parameters $\left(n_{j}, k_{j}\right.$, $\left.\gamma_{j} ; m_{j},\left(d_{f}\right)_{j}\right)_{q}$ is said to be asymptotically good if $\limsup _{j \rightarrow \infty} k_{j} / n_{j}>0$ and $\limsup _{j \rightarrow \infty}\left(d_{f}\right)_{j} / r_{j}>0$ hold, where $s_{j}:=\left(n_{j}-k_{j}\right)\left[\left\lfloor\gamma_{j} / k_{j}\right\rfloor+1\right]+\gamma_{j}+1$ and $r_{j}:=\max \left\{n_{j}, s_{j}\right\}$.

Remark 3.1 Note that because both sequences of real numbers $\left(k_{j} / n_{j}\right)_{j \geq 0}$ and $\left(\left(d_{f}\right)_{j} / r_{j}\right)_{j \geq 0}$ are bounded, then there exist both $\lim \sup _{j \rightarrow \infty} k_{j} / n_{j}$ and $\lim _{\sup _{j \rightarrow \infty}}$ $\left(d_{f}\right)_{j} / r_{j}$, and the definition makes sense.

Let us recall the construction of a sequence of asymptotically good transitive codes:

Theorem 3.2 [35, Theorem 1.5] Let $q=l^{2}$ be a square. Then the class of transitive codes meets the Tsfasman-Vladut-Zink bound. More precisely, let $R, \delta \geq 0$ be real numbers with $R=1-\delta-1 /(l-1)$. Then there exists a sequence $\left(C_{j}\right)_{j>0}$ of linear codes $C_{j}$ over $\mathbb{F}_{q}$ with parameters $\left[n_{j}, k_{j}, d_{j}\right]_{q}$ with the following properties:

a) All $C_{j}$ are transitive codes.

b) $n_{j} \rightarrow \infty$ as $j \rightarrow \infty$.

c) $\lim _{j \rightarrow \infty} k_{j} / n_{j} \geq R$ and $\lim _{j \rightarrow \infty} d_{j} / n_{j} \geq \delta$.

The codes shown in Theorem 3.2 are AG codes constructed by applying an asymptotically good tower of function fields over $\mathbb{F}_{q}$ (see [35]). For more details with respect to AG codes, see [36].

In this paper we only construct sequences of unit-memory convolutional codes. Moreover, we construct reduced basic generator matrices for the new asymptotically good sequences of convolutional codes, i.e., the codes are noncatastrophic.

Theorem 3.3 Let $q=l^{2}$ be a prime power, where $l \geq 3$ is an integer. Then there exists a sequence of asymptotically good convolutional codes, over $\mathbb{F}_{q}$, derived from transitive codes.

Proof: We adopt the same notation of Theorem 3.2. Let $R>0$ and $\delta>0$ be real numbers with $R=1-\delta-1 /(l-1)$. Since $\lim _{j \rightarrow \infty} 1 / n_{j}=0$ and $\lim _{j \rightarrow \infty} k_{j} / n_{j} \geq R>0$, then it follows that the sequence $\left(k_{j}\right)_{j>0}$ of positive integers is not bounded. Taking $c=1$, there exists a nonnegative integer $j_{1}$ such that $k_{j_{1}}>1$. Analogously, there exists a positive integer $j_{2}>j_{1}$ such that $k_{j_{2}}>k_{j_{1}}$, otherwise $\left(k_{j}\right)_{j>0}$ would be bounded. Assume by induction that we have defined the subsequence for $n$ numbers $j_{1}<j_{2}<\ldots<j_{n}$, i.e., $1<k_{j_{1}}<k_{j_{2}}<\ldots<k_{j_{n}}$, where $j_{1}<j_{2}<\ldots<j_{n}$. Then there exists a positive integer $j_{n+1}>j_{n}$ such that $k_{j_{n+1}}>k_{j_{n}}$, otherwise $\left(k_{j}\right)_{j \geq 0}$ would 
be bounded. Thus we extract a subsequence $\left(k_{j_{t}}\right)_{t \geq 0}$ of $\left(k_{j}\right)_{j \geq 0}$ with $k_{j_{t}}>1$ for each $t \geq 0$ and $k_{j_{t}} \rightarrow \infty$ as $t \rightarrow \infty$. Moreover, it is clear that $n_{j_{t}} \rightarrow \infty$ as $t \rightarrow \infty$ (alternatively, because $\lim _{j \rightarrow \infty} k_{j} / n_{j}>0$, and since $n_{j} \rightarrow \infty$ when $j \rightarrow \infty$, there exists a positive integer $n_{0}$ such that, $\forall j>n_{0}$, one has $k_{j}>1$ ).

We first consider the (Euclidean) dual $C_{j_{t}}^{\perp}$ of the transitive code $C_{j_{t}}$ constructed in Theorem 3.2. for every $t \geq 0$. To simplify the notation we put $C_{j_{t}}:=C_{t}$, for all $t \geq 0$. A parity check matrix $G_{t}$ of $C_{t}^{\perp}$ is a generator matrix of $C_{t}$, for all $t \geq 0$. We construct a sequence of convolutional codes $V_{t}$, for all $t \geq 0$, generated the by reduced basic matrix

$$
\mathbb{G}_{t}(D)=G_{t}^{*}+\tilde{L}_{t} D,
$$

where $G_{t}^{*}$ is the submatrix of $G_{t}$ consisting of the first $k_{t}-1$ rows of $G_{t}$ and $\tilde{L}_{t}$ is the matrix consisting of the last row of $G_{t}$ together $k_{t}-2$ zerorows at the bottom. The code $V_{t}$ is a unit-memory code with parameters $\left(n_{t}, k_{t}-1,1 ; 1,\left(d_{f}\right)_{t}\right)_{q}, t \geq 0$. From Theorem 2.1] one has $\left(d_{f}\right)_{t} \geq d_{t}$. It is obvious that $\lim _{t \rightarrow \infty}\left(k_{t}-1\right) / n_{t} \geq R>0$. On the other hand, since $s_{t}=$ $n_{t}-k_{t}+2$ and because $k_{t}>1$ for all $t \geq 0$, then $r_{t}=n_{t}$; thus it follows that $\lim _{t \rightarrow \infty}\left(d_{f}\right)_{t} / r_{t}>0$. Therefore, we have constructed an asymptotically good sequence $\left(V_{t}\right)_{t \geq 0}$ of convolutional codes, as desired.

Theorem 3.4 Let $q=l^{2}$ be a prime power, where $l \geq 3$ is an integer. Then, for any positive integer $\gamma_{0}>1$ there exists a sequence of asymptotically good convolutional codes, over $\mathbb{F}_{q}$, with degree $\gamma=\gamma_{0}$.

Proof: By the same reasoning utilized in the first part of the proof of Theorem [3.3 we construct a subsequence $\left(k_{t}\right)_{t>0}$ of $\left(k_{j}\right)_{j>0}$ such that $k_{t} \rightarrow \infty$ as $t \rightarrow \infty$ with $\gamma_{0}<k_{1}<k_{2}<\ldots<k_{t}<\ldots$, where $n=1,2,3, \ldots$. Additionally, it is clear that $n_{t} \rightarrow \infty$ as $t \rightarrow \infty$ (alternatively, proceeding similarly as in the proof of Theorem 3.3. we show the existence of a positive integer $n_{0}$ such that, for all $j>n_{0}$, one has $k_{j}>\gamma_{0}$ ). We then take the dual $C_{t}^{\perp}$ of $C_{t}$, for every $t \geq 0$ and consider a parity check matrix $G_{t}$ of $C_{t}^{\perp}$ which is a generator matrix of $C_{t}$. We construct a sequence of convolutional codes $V_{t}$, for all $t \geq 0$, generated by the reduced basic matrix $\mathbb{G}_{t}(D)=G_{t}^{*}+\tilde{L}_{t} D$, where $G_{t}^{*}$ is the submatrix of $G_{t}$ consisting of the first $k_{t}-\gamma_{0}$ rows of $G_{t}$ and $\tilde{L}_{t}$ is the matrix consisting of the last $\gamma_{0}$ rows of $G_{t}$ together $k_{t}-2 \gamma_{0}$ zero-rows at the bottom. The code $V_{t}$ is a unit-memory code with parameters $\left(n_{t}, k_{t}-\gamma_{0}, \gamma_{0} ; 1,\left(d_{f}\right)_{t}\right)_{q}, t \geq 0$. We know that $s_{t}=n_{t}-k_{t}+\gamma_{0}+1$ and $r_{t}=n_{t}$. Since $\lim _{t \rightarrow \infty}\left(k_{t}-\gamma_{0}\right) / n_{t}>0$ and $\lim _{t \rightarrow \infty}\left(d_{f}\right)_{t} / r_{t}>0$ are true, the result follows.

We next construct new families of asymptotically good convolutional codes obtained by expanding, extending, puncturing, direct sum, the $\langle u \mid u+v\rangle$ construction and by the product code construction applied to the sequence of transitive codes shown in Theorem 3.2. For more details concerning such techniques of construction, see [23, 12]. 
From now on, to simplify the notation, we always consider the existence of a (sub)sequence $\left(C_{j}\right)_{j \geq 0}$ of linear codes with parameters $\left[n_{j}, k_{j}, d_{j}\right]_{q}$, constructed in Theorem 3.2, such that $k_{j}>1$ (or $k_{j}>\gamma_{0}$, for all $\gamma_{0} \geq 1$, according to Theorem 3.4) for all $j \geq 0$.

Theorem 3.5 Let $q^{m}=l^{2}$ be a prime power, where $l \geq 3$ and $m>1$ are integers. Then there exists a sequence of asymptotically good convolutional codes, over $\mathbb{F}_{q}$, derived from expansion of transitive codes.

Proof: Let $R>0$ and $\delta>0$ be real numbers with $R=1-\delta-1 /(l-1)$. Consider a (sub)sequence $\left(C_{j}\right)_{j \geq 0}$ of asymptotically good linear codes $C_{j}$, over $\mathbb{F}_{q^{m}}$, with parameters $\left[n_{j}, k_{j}, d_{j}\right]_{q^{m}}$, shown in Theorem 3.2 Let $\beta=\left\{b_{1}, b_{2}, \ldots, b_{m}\right\}$ be a basis of $\mathbb{F}_{q^{m}}$ over $\mathbb{F}_{q}$. We expand all codes $C_{j}$ with respect to $\beta$ generating codes $\beta\left(C_{j}\right)$, over $\mathbb{F}_{q}$, with parameters $\left[m n_{j}, m k_{j}, d_{j}^{*} \geq d_{j}\right]_{q}$, for all $j \geq 0$. Consider the dual $\left[\beta\left(C_{j}\right)\right]^{\perp}$ of the code $\beta\left(C_{j}\right)$, for all $j \geq 0$. A parity check matrix $G_{j}$ of $\left[\beta\left(C_{j}\right)\right]^{\perp}$ is a generator matrix of $\beta\left(C_{j}\right)$, for all $j \geq 0$. Proceeding similarly as in the proof of Theorem 3.3, the result follows.

Let $V_{j}$ be the convolutional code generated by the reduced basic matrix $\mathbb{G}_{j}(D)=G_{j}^{*}+\tilde{L}_{j} D$, where $G_{j}^{*}$ is the submatrix of $G_{j}$ consisting of the $k_{j}-1$ first rows of $G_{j}$ and $\tilde{L}_{j}$ is the matrix consisting of the last row of $G_{j}$ together $k_{j}-2$ zero-rows at the bottom, for all $j \geq 0$. $V_{j}$ has parameters $\left(m n_{j}, m k_{j}-\right.$ 1,$\left.1 ; 1,\left(d_{f}\right)_{j}\right)_{q}$, where $\left(d_{f}\right)_{j} \geq d_{j}^{*} \geq d_{j}$. We know that $\lim _{j \rightarrow \infty}\left(m k_{j}-1\right) / m n_{j} \geq$ $R>0$. On the other hand, one has $s_{j}=m\left(n_{j}-k_{j}\right)+3$ and $r_{j} \leq m n_{j}+1$. Thus, it follows that $\left(d_{f}\right)_{j} / r_{j} \geq\left(d_{f}\right)_{j} /\left(m n_{j}+1\right) \geq d_{j} /\left(m n_{j}+1\right)$. Because $\lim _{j \rightarrow \infty} d_{j} /\left(m n_{j}+1\right)=[1 / m] \lim _{j \rightarrow \infty} d_{j} / n_{j}$, we conclude that $\lim _{j \rightarrow \infty}\left(d_{f}\right)_{j} / r_{j}>$ 0 . Thus, the sequence $\left(V_{j}\right)_{j \geq 0}$ of convolutional codes is asymptotically good and the proof is complete.

Applying the techniques of combining codes, we can get more sequences of good convolutional codes.

Theorem 3.6 Let $q=l^{2}$ be a prime power, where $l \geq 3$ is an integer. Then there exists a sequence of asymptotically good convolutional codes, over $\mathbb{F}_{q}$, derived from direct sum of transitive codes.

Proof: Consider a (sub)sequence of codes $\left(C_{j}\right)_{j \geq 0}$ shown in Theorem 3.2 with $k_{j}>1$ for all $j \geq 0$. We construct the sequence of direct sum codes $C_{j} \oplus C_{j}$ with parameters $\left[2 n_{j}, 2 k_{j}, d_{j}\right]_{q}$, for all $j \geq 0$. Let $G_{j}$ be a generator matrix of the code $C_{j} \oplus C_{j}$, for all $j \geq 0$. Consider the dual $\left[C_{j} \oplus C_{j}\right]^{\perp}$ of the code $C_{j} \oplus C_{j}$, $j \geq 0 ; G_{j}$ is a parity check matrix for $\left[C_{j} \oplus C_{j}\right]^{\perp}$.

Let $V_{j}$ be the convolutional code generated, by the reduced basic matrix $\mathbb{G}_{j}(D)=G_{j}^{*}+\tilde{L}_{j} D$, where $G_{j}^{*}$ is the submatrix of $G_{j}$ consisting of the $k_{j}-1$ first rows of $G_{j}$ and $\tilde{L}_{j}$ is the matrix consisting of the last row of $G_{j}$ together $k_{j}-2$ zero-rows at the bottom, for all $j \geq 0$. $V_{j}$ has parameters $\left(2 n_{j}, 2 k_{j}-1,1 ; 1,\left(d_{f}\right)_{j}\right)_{q}$, where $\left(d_{f}\right)_{j} \geq d_{j}$. We know that $s_{j}=2\left(n_{j}-k_{j}\right)+3$ 
and $r_{j} \leq 2 n_{j}+1$, for all $j \geq 0$, the free distance $\left(d_{f}\right)_{j}$ of the code $V_{j}$ satisfies $\left(d_{f}\right)_{j} / r_{j} \geq d_{j} /\left(2 n_{j}+1\right)$. Because $\lim _{j \rightarrow \infty} d_{j} /\left(2 n_{j}+1\right)=[1 / 2] \lim _{j \rightarrow \infty} d_{j} / n_{j}$, it follows that $\lim _{j \rightarrow \infty}\left(d_{f}\right)_{j} / r_{j}>0$. On the other hand, $\lim _{j \rightarrow \infty}\left(2 k_{j}-1\right) / 2 n_{j}=$ $\lim _{j \rightarrow \infty} k_{j} / n_{j}>0$. Therefore, the sequence $\left(V_{j}\right)_{j \geq 0}$ of convolutional codes is asymptotically good.

Theorem 3.7 Let $q=l^{2}$ be a prime power, where $l \geq 3$ is an integer. Then there exists a sequence of asymptotically good convolutional codes, over $\mathbb{F}_{q}$, derived from the $\langle u \mid u+v\rangle$ construction of transitive codes.

Proof: The proof is similar to that of Theorem 3.6.

In Theorems 3.8 and 3.9, we apply code extension and puncturing of transitive codes, respectively, in order to construct more two sequences of asymptotically good codes.

Theorem 3.8 Let $q=l^{2}$ be a prime power, where $l \geq 3$ is an integer. Then there exists a sequence of asymptotically good convolutional codes, over $\mathbb{F}_{q}$, derived from extension of transitive codes.

Proof: Assume that $\left(C_{j}\right)_{j \geq 0}$ is a (sub)sequence of codes shown in Theorem 3.2 with $k_{j}>1$ for all $j>0$. We construct a sequence $\left(C_{j}^{e}\right)_{j>0}$ of codes with parameters $\left[n_{j}+1, k_{j}, d_{j}^{e}\right]_{q}$, where $d_{j}^{e}=d_{j}$ or $d_{j}^{e}=d_{j}+1$. The sequence of convolutional codes is constructed similarly as in the proofs of Theorems 3.3 to 3.6. We know that $\lim _{j \rightarrow \infty}\left(k_{j}-1\right) /\left(n_{j}+1\right)=\lim _{j \rightarrow \infty} k_{j} / n_{j}>0$ and that $s_{j}=n_{j}-k_{j}+4$. Hence, $\lim _{j \rightarrow \infty}\left(d_{f}\right)_{j} / r_{j} \geq \lim _{j \rightarrow \infty} d_{j} / r_{j}>0$. The proof is complete.

Theorem 3.9 Let $q=l^{2}$ be a prime power, where $l \geq 3$ is an integer. Then there exists a sequence of asymptotically good convolutional codes, over $\mathbb{F}_{q}$, derived from puncturing transitive codes.

Proof: Let $\left(C_{j}\right)_{j \geq 0}$ be a (sub)sequence of codes shown in Theorem 3.2 with $k_{j}>1$ for all $j>0$. By puncturing the codes $C_{j}, j \geq 0$, in the $i$ th coordinate, we construct a new sequence $\left(C_{j}^{p}\right)_{j \geq 0}$ of codes with parameters $\left[n_{j}-1, k_{j}, d_{j}^{p}\right]_{q}$, where $d_{j}^{p}=d_{j}-1$ if $C_{j}$ has a minimum weight codeword with a nonzero $i$ th coordinate and $d_{j}^{p}=d_{j}$ otherwise. Note that we can assume w.l.o.g. that $d_{j}>1$ for all $j \geq 0$; see the first part of the proof of Theorem 3.3 applied to $d_{j}$ instead of $k_{j}$. We construct a sequence of convolutional codes as in Theorems 3.3 to 3.6. The sequence $\left(V_{j}\right)_{j \geq 0}$ consists of convolutional codes with parameters $\left(n_{j}-1, k_{j}-1,1 ; 1,\left(d_{f}\right)_{j}\right)_{q}$, where $\left(d_{f}\right)_{j} \geq d_{j}-1$ and $s_{j}=n_{j}-k_{j}+2$. It is clear that $\lim _{j \rightarrow \infty}\left(k_{j}-1\right) /\left(n_{j}-1\right)>0$ and $\lim _{j \rightarrow \infty}\left(d_{f}\right)_{j} / r_{j}>0$. Therefore, $\left(V_{j}\right)_{j \geq 0}$ is asymptotically good and we are done. 
Before showing Theorem 3.10 , we must recall the direct product construction of linear (block) codes. For more details, see [23].

Definition 3.2 [23] Let $C_{1}=\left[n_{1}, k_{1}, d_{1}\right]_{q}$ and $C_{2}=\left[n_{2}, k_{2}, d_{2}\right]_{q}$ be linear codes both over $\mathbb{F}_{q}$, with generator matrices $G^{(1)}$ and $G^{(2)}$, respectively. Then the product code $C_{\pi}:=C_{1} \otimes C_{2}$ is a linear code, over $\mathbb{F}_{q}$, with parameters $C_{\pi}=$ $\left[n_{1} n_{2}, k_{1} k_{2}, d_{1} d_{2}\right]_{q}$, generated by the matrix $G^{(\pi)}=G^{(1)} \otimes G^{(2)}$, where $\otimes$ denotes the Kronecker product of matrices, that is,

$$
G^{(\pi)}=\left[\begin{array}{cccc}
g_{11}^{(1)} G^{(2)} & g_{12}^{(1)} G^{(2)} & \cdots & g_{1 n_{1}}^{(1)} G^{(2)} \\
g_{21}^{(1)} G^{(2)} & g_{22}^{(1)} G^{(2)} & \cdots & g_{2 n_{1}}^{(1)} G^{(2)} \\
\vdots & \vdots & \vdots & \vdots \\
g_{k_{1} 1}^{(1)} G^{(2)} & g_{k_{1} 2}^{(1)} G^{(2)} & \cdots & g_{k_{1} n_{1}}^{(1)} G^{(2)}
\end{array}\right]
$$

where $g_{i j}^{(t)}, t=1,2$, is the ij-th entry of the matrix $G^{(t)}$, respectively.

As we will see in Theorem 3.10 given in the following, direct product codes obtained from transitive codes are also asymptotically good.

Theorem 3.10 Let $q=l^{2}$ be a prime power, where $l \geq 3$ is an integer. Then there exists a sequence of asymptotically good convolutional codes, over $\mathbb{F}_{q}$, derived from direct product of transitive codes.

Proof: Let $\left(C_{j}\right)_{j \geq 0}$ be a (sub)sequence of codes shown in Theorem 3.2 with $k_{j}>1$ for all $j>0$. Let us consider the sequence of product codes $\left(C_{j} \otimes C_{j}\right)_{j \geq 0}$. This sequence consists of codes with parameters $\left[\left(n_{j}\right)^{2},\left(k_{j}\right)^{2},\left(d_{j}\right)^{2}\right]_{q}$. Constructing the sequence $\left(V_{j}\right)_{j \geq 0}$ of convolutional codes similarly as in the proof of Theorems 3.3 to 3.9 , we obtain codes with parameters $\left(\left(n_{j}\right)^{2},\left(k_{j}\right)^{2}-1,1 ; 1,\left(d_{f}\right)_{j}\right)_{q}$, with $\left(d_{f}\right)_{j} \geq\left(d_{j}\right)^{2}$, where $s_{j}=\left(n_{j}\right)^{2}-\left(k_{j}\right)^{2}+3$. It is straightforward to see that $\lim _{j \rightarrow \infty}\left[\left(k_{j}\right)^{2}-1\right] /\left(n_{j}\right)^{2}>0$ and $\lim _{j \rightarrow \infty}\left(d_{f}\right)_{j} / r_{j}>0$ are true. Thus, the result follows.

Let $C$ be a linear code. Recall that $C$ is called self-orthogonal if $C \subset C^{\perp}$, and $C$ is called self-dual if $C=C^{\perp}$, where $C^{\perp}$ is the (Euclidean) dual of the code $C$. The following theorem will be also utilized to construct more sequences of asymptotically good convolutional codes:

Theorem 3.11 [35, Theorem 1.6] Let $q=l^{2}$ be a square. Then the class of selforthogonal codes and the class of self-dual codes meets the Tsfasman-Vladut-Zink bound. More precisely, the following are true:

(i) Let $0 \leq R \leq 1 / 2$ and $\delta \geq 0$ be real numbers with $R=1-\delta-1 /(l-1)$. Then there exists a sequence $\left(C_{j}\right)_{j \geq 0}$ of linear codes $C_{j}$ over $\mathbb{F}_{q}$ with parameters $\left[n_{j}, k_{j}, d_{j}\right]_{q}$ such that:

a) All $C_{j}$ are self-orthogonal codes. 
b) $n_{j} \rightarrow \infty$ as $j \rightarrow \infty$.

c) $\lim _{j \rightarrow \infty} k_{j} / n_{j} \geq R$ and $\lim _{j \rightarrow \infty} d_{j} / n_{j} \geq \delta$.

(ii) There exists a sequence $\left(D_{j}\right)_{j \geq 0}$ of self-dual codes $D_{j}$ over $\mathbb{F}_{q}$ with parameters $\left[n_{j}, n_{j} / 2, d_{j}\right]_{q}$ such that $n_{j} \rightarrow \infty$ and $\lim _{j \rightarrow \infty} d_{j} / n_{j} \geq 1 / 2-1 /(l-1)$.

Theorem 3.12 Let $q=l^{2}$ be a prime power, where $l \geq 3$ is an integer. Consider the two sequences $\left(C_{j}\right)_{j \geq 0}$ and $\left(D_{j}\right)_{j \geq 0}$ of self-orthogonal and self-dual linear codes respectively, over $\mathbb{F}_{q}$ shown in Theorem 3.11. Then the following hold

(i) There exist two sequences of asymptotically good convolutional codes $\left(V_{j}\right)_{j \geq 0}$ and $\left(W_{j}\right)_{j \geq 0}$, derived respectively from $\left(C_{j}\right)_{j \geq 0}$ and $\left(D_{j}\right)_{j \geq 0}$.

(ii) There exist sequences of asymptotically good convolutional codes $\left(V_{j}^{k}\right)_{j>0}$ derived, respectively, from $\left(C_{j}^{k}\right)_{j \geq 0}, k=1, \ldots 6$, where the codes $C_{j}^{1}, C_{j}^{2}$, $\ldots, C_{j}^{6}$ are obtained from $\left(C_{j}\right)_{j \geq 0}$ by expanding, direct sum, the $\langle u \mid u+v\rangle$ construction, extending, puncturing and from direct product construction, respectively.

(iii) There exist sequences of asymptotically good convolutional codes $\left(W_{j}^{k}\right)_{j \geq 0}$ derived, respectively, from $\left(D_{j}^{k}\right)_{j \geq 0}, k=1, \ldots 6$, where the codes $D_{j}^{1}, D_{j}^{2}$, $\ldots, D_{j}^{6}$ are obtained from $\left(D_{j}\right)_{j \geq 0}$ by expanding, direct sum, the $\langle u \mid u+v\rangle$ construction, extending, puncturing and from direct product construction, respectively.

Proof: The proof is similar to that proofs of Theorems 3.3 to 3.10 respectively.

Let us recall the definition of asymptotically good block codes:

Definition 3.3 A sequence $\left(C_{j}\right)_{j \geq 0}$ of linear codes over $\mathbb{F}_{q}$ with parameters $\left[n_{j}\right.$, $\left.k_{j}, d_{j}\right]_{q}$ is said to be asymptotically good if $n_{j} \rightarrow \infty$ as $j \rightarrow \infty$ and if $\lim \sup _{j \rightarrow \infty}$ $k_{j} / n_{j}>0$ and $\lim \sup _{j \rightarrow \infty} d_{j} / n_{j}>0$ hold.

Theorem 3.13, given in the following, establishes that the results presented in this paper are also true in a more general scenario, when considering arbitrary families of asymptotically good linear block codes in order to construct AGCC's:

Theorem 3.13 Let $q$ be a prime power. Assume that there exists a sequence $\left(C_{j}\right)_{j \geq 0}$ of asymptotically good linear (block) codes $C_{j}$, where both limits $\lim _{j \rightarrow \infty}$ $k_{j} / n_{j}$ and $\lim _{j \rightarrow \infty} d_{j} / n_{j}$ exist. Then the following hold:

(i) There exists a sequence of asymptotically good convolutional codes $\left(V_{j}\right)_{j \geq 0}$ derived from $\left(C_{j}\right)_{j \geq 0}$. 
(ii) There exist sequences of asymptotically good convolutional codes $\left(V_{j}^{k}\right)_{j \geq 0}$, $k=1, \ldots 6$, derived, respectively, from the sequences $\left(C_{j}^{k}\right)_{j \geq 0}$ of linear codes, $k=1, \ldots 6$, where the codes $C_{j}^{1}, C_{j}^{2}, \ldots, C_{j}^{6}$ are obtained from $\left(C_{j}\right)_{j \geq 0}$ by expanding, direct sum, the $\langle u \mid u+v\rangle$ construction, extending, puncturing and from direct product construction, respectively.

Proof: The proof is similar to that proofs of Theorems 3.3 to 3.10 respectively.

\section{Summary}

We have constructed new sequences of asymptotically good convolutional codes derived from sequences of transitive, self-orthogonal and self-dual block codes that attain the Tsfasman-Vladut-Zink bound. Furthermore, more sequences of new asymptotically good convolutional codes were obtained by applying the techniques of expanding, extending, puncturing, direct sum, the $\langle u \mid u+v\rangle$ construction and the product code construction to these block codes. Additionally, we have shown that our new constructions of AGCC's also hold when applied to all sequences of asymptotically good block codes where $\lim _{j \rightarrow \infty} k_{j} / n_{j}$ and $\lim _{j \rightarrow \infty} d_{j} / n_{j}$ exist.

\section{Acknowledgment}

I would like to thank the anonymous referee for his/her valuable comments and suggestions that improve significantly the quality and the presentation of this paper. This research has been partially supported by the Brazilian Agencies CAPES and CNPq.

\section{References}

[1] S.A. Aly, M. Grassl, A. Klappenecker, M. Rötteler, P.K. Sarvepalli. Quantum convolutional BCH codes. In Proc. Canadian Workshop on Information Theory (CWIT), pp.180-183, 2007.

[2] I.E. Bocharova, B.D. Kudryashov and R. Johannesson. Searching for binary and nonbinary block and convolutional LDPC codes. IEEE Trans. Inform. Theory, 62(1):163-183, 2016.

[3] Z.G. Chen and S. Bates. Construction of low-density parity-check convolutional codes through progressive edge-growth. IEEE Commun Lett, 9:1058-1060, 2005.

[4] A.J. Felstrom and K.Sh. Zigangirov. Time-varying periodic convolutional codes with low-density parity-check matrices. IEEE Trans. Inform. Theory, 45(6):21812191, 1999.

[5] G.D. Forney Jr. Convolutional codes I: algebraic structure. IEEE Trans. Inform. Theory, 16(6):720-738, 1970. 
[6] A. Garcia, H. Stichtenoth. A tower of Artin-Schreier extensions of function fields attaining the Drinfeld-Vladut bound. Invent. Math., 121:211-222, 1995.

[7] A. Garcia, H. Stichtenoth. On the asymptotic behaviour of some towers of function fields over finite fields. J. Number Theory, 61:248-273, 1996.

[8] H. Gluesing-Luerssen, J. Rosenthal, R. Smarandache. Strongly MDS convolutional codes. IEEE Trans. Inform. Theory, 52:584-598, 2006.

[9] H. Gluesing-Luerssen, W. Schmale. Distance bounds for convolutional codes and some optimal codes. e-print arXiv:math/0305135.

[10] V.D. Goppa. A new class of linear correcting codes. Probl. Peredachi Inf., (6):2430, 1970.

[11] V.D. Goppa. Codes on algebraic curves. Soviet Math. Dokl, 22(1):170-172, 1981.

[12] W.C. Huffman, V. Pless. Fundamentals of Error Correcting Codes. Cambridge Univ. Press, 2003.

[13] R. Hutchinson, J. Rosenthal, R. Smarandache. Convolutional codes with maximum distance profile. Systems and Control Letters, 54(1):53-63, 2005.

[14] J.I. Iglesias-Curto. Generalized AG convolutional codes. Advances in Mathematics of Communications, 3(4):317-328, 2009.

[15] R. Johannesson, K.S. Zigangirov. Fundamentals of Convolutional Coding. Digital and Mobile Communication, Wiley-IEEE Press, 1999.

[16] J. Justesen. New convolutional code constructions and a class of asymptotically good time-varying codes. IEEE Trans. Inform. Theory, 19(2):220-225, 1973.

[17] M. Kuijper, R. Pinto. On Minimality of convolutional ring encoders. IEEE Trans. Inform. Theory, 55(11):4890-4897, 2009.

[18] G.G. La Guardia. On classical and quantum MDS-convolutional BCH codes. IEEE Trans. Inform. Theory, 60(1):304-312, 2014.

[19] G.G. La Guardia. On negacyclic MDS-convolutional codes. Linear Alg. Applic., 448:85-96, 2014.

[20] G.G. La Guardia. On optimal constacyclic codes. Linear Alg. Applic., 496:594610, 2016.

[21] M. Lentmaiert, D.G.M. Mitchell, G. Fettweist, D.J. Costello, Jr. Asymptotically good LDPC convolutional codes with AWGN channel thresholds close to the Shannon limit. In 6th Int. Symp. on Turbo Codes and Iterative Inform. Processing, pp:324-328, 2010.

[22] D.J.C. MacKay, M.S. Postol. Weaknesses of Margulis and Ramanujan-Margulis low-density parity-check codes. Electron. Notes Theoretical Comput. Sci., 74:97104, 2003.

[23] F.J. MacWilliams, N.J.A. Sloane. The Theory of Error-Correcting Codes. NorthHolland, 1977.

[24] D.G.M. Mitchell, A.E. Pusane, K.Sh. Zigangirov, D.J. Costello Jr.. Asymptotically good LDPC convolutional codes based on protographs. In Proc. Int. Symp. Inform. Theory (ISIT), pp.1030-1034, 2008.

[25] D.G.M. Mitchell, M. Lentmaier, D.J. Costello Jr.. Spatially coupled LDPC codes constructed from protographs. IEEE Trans. Inform. Theory, 61(9):4866-4889, 2015 . 
[26] D.G.M. Mitchell, A.E. Pusane, D.J. Costello Jr.. Minimum distance and trapping set analysis of protograph-based LDPC convolutional codes. IEEE Trans. Inform. Theory, 59(1):254-281, 2013.

[27] L. Mu, X. Liu and C. Liang. Improved construction of LDPC convolutional codes with semi-random parity-check matrices. Science China, 57:022304:1-022304:10, 2014.

[28] D. Napp, R. Pinto, M. Toste. On MDS convolutional codes over $\mathbb{Z}_{p}^{r}$. Designs, Codes and Cryptography, 83(1):101-114, 2017.

[29] P.D. Papadimitriou, C.N. Georghiades. On asymptotically optimum rate $1 / n$ convolutional codes for a given constraint length. IEEE Commun. Letters, 5(1):25-27, 2001.

[30] Ph Piret. Phillips Res. Report, (27):257-271, 1972.

[31] Ph. Piret. Convolutional Codes: An Algebraic Approach. Cambridge, Massachusetts: The MIT Press, 1988.

[32] J. Rosenthal, R. Smarandache. Maximum distance separable convolutional codes. Applicable Algebra in Eng. Comm. Comput., 10:15-32, 1999.

[33] R. Smarandache, H. G.-Luerssen, J. Rosenthal. Constructions of MDSconvolutional codes. IEEE Trans. Inform. Theory, 47(5):2045-2049, 2001.

[34] A. Sridharan, D. Truhachev, M. Lentmaier, D.J. Costello Jr., K.Sh. Zigangirov. Distance bounds for an ensemble of LDPC convolutional codes. IEEE Trans. Inform. Theory, 53(12):4537-4555, 2007.

[35] H. Stichtenoth. Transitive and self-dual codes attaining the Tsfasman-VladutZink bound. IEEE Trans. Inform. Theory, 52(5):2218-2224, 2006.

[36] H. Stichtenoth. Algebraic Function Fields and Codes. Springer, 2009.

[37] H. Uchikawa, K. Kasai and K. Sakaniwa. Design and performance of ratecompatible non-binary LDPC convolutional codes. IEICE Trans. on Fundamentals of Electronics, Communications and Computer Sciences, E94.A(11):21352143, 2011. 